\title{
Analytical impact of Metschnikowia pulcherrima in the volatile profile of Verdejo white wines
}

\author{
Javier Ruiz • Ignacio Belda • Beata Beisert • Eva Navascués · Domingo Marquina · Fernando Calderón • \\ Doris Rauhut · Antonio Santos · Santiago Benito
}

\begin{abstract}
Most wine aroma compounds, including the varietal fraction, are produced or released during wine production and derived from microbial activity. Varietal aromas, typically defined as terpenes and thiols, have been described as derived from their non-volatile precursors, released during wine fermentation by different yeast hydrolytic enzymes. The perception of these minority aroma compounds depends on the chemical matrix of the wine, especially on the presence of majority aroma compounds, such as esters or higher alcohols. Strategies aiming to reduce the production of these masking flavors are on the spotlight of enology research as a way to better define varietal standard profiles for the global market. Using a natural white must from Verdejo variety (defined as a thiol grape variety), here we describe the analytical and sensorial impact of using, in sequential inoculations, a selected strain of Metschnikowia pulcherrima, in combination with two different Saccharomyces cerevisiae strains. An increase in the levels of the thiol 4-MSP (4-methyl-4-sulfanylpentan-2-one) over its sensory threshold, together with a decrease in higher alcohol production, was observed when $M$. pulcherrima was used. This has an important impact on these wines, making them fruitier and fresher, always preferred by the sensory panel.
\end{abstract}

\section{Introduction}

The chemistry explaining wine flavor has been widely studied due to its abnormal complexity. The role of yeasts in wine production, turning grape must into wine, exceeds the mere conversion of sugars into ethanol since a great diversity of metabolic pathways (coming from a great microbial diversity associated to wine fermentation) are involved in the production of thousands of volatile and non-volatile compounds comprising the final sensory properties of a wine (Belda et al. 2017a). While the preferences of average customers looking for red wines are on the balance of wood-related compounds and fermentative aromas (such as certain esters and higher alcohols), in the case of white wines, the global market is looking for the empowering of varietal (fruity, floral, and fresh) aromas.

The sensory profile of many wines depends on the presence and release of varietal compounds (especially in the case of white wines, where post-fermentative aging practices are scarcer or subtler than in red wines). These varietal 
compounds can be present in grapes as free volatile compounds and. in much higher concentrations, as aroma precursors which can be naturally and slowly released during wine aging or intentionally released during winemaking by using oenological enzymes or yeast strains with specific hydrolytic activities (Baumes 2009). Terpenes and thiols are the main drivers of the 'varietal' fraction of wine aroma, and they tend to appear conjugated with sugars (xylose, rhamnose. arabinose, apiose) and amino acids (cysteine and glutathione), respectively. Both terpenes and polyfunctional thiols are highimpact aroma compounds, with a strong influence in wine sensorial properties because of their low detection thresholds, despite their low concentration in wines (Mateo and Jiménez 2000: Tominaga et al. 1998). However. it is necessary to understand the synergist-antagonist effects in the presence of other volatile compounds found in a wine matrix. where high levels of higher alcohols or certain esters can overshadow the sensory impact of the mentioned minority compounds.

Polyfunctional thiols (commonly known as 'tropical thiols' in wine) are, together with terpenes, among the main volatile compounds contributing to the varietal fraction of wine aroma, defining the sensorial characteristics of certain white wine varieties, such as Sauvignon blanc and Verdejo. Mainly, polyfunctional thiols in wines include 4-methyl-4sulfanylpentan-2-one (4-MSP), 3-sulfanylhexan-1-ol (3-SH). and its acetylated derivative 3 -sulfanylhexyl acetate (3-SHA). Their substantial contribution to wine aroma can be justified because of their very low detection thresholds: $3 \mathrm{ng} / \mathrm{L}$ (4-MSP). $60 \mathrm{ng} / \mathrm{L}$ (3-SH), and $4 \mathrm{ng} / \mathrm{L}$ (3-SHA) (Swiegers et al. 2005).

The presence of 4-MSP and 3-SH in grapes and musts is, predominantly, in a conjugated non-volatile way. They used to be bounded with cysteine or glutathione (Fedrizzi et al. 2009; Tominaga et al. 1995), and thus, a carbon-sulfur lyase activity (mainly produced by certain wine yeasts) is necessary for an enzymatic cleave of the conjugated precursor, releasing the correspondent volatile thiols (Dubourdieu et al. 2006). In this context, the enzymatic properties of the yeast strain used in a wine fermentation should be taken into account when it is desired to enhance the varietal aroma of a wine (Belda et al. 2017b).

$\beta$-Lyase activity, as the main activity related to thiol release, appeared to be scarce in most wine-related yeast species. but also with a remarkable strain-dependent behavior (Belda et al. 2016a: Zott et al. 2011). However, a few nonSaccharomyces species stand out because of their $\beta$-lyase activity (Belda et al. 2016b). Among them, some Metschnikowia strains stand out because of their $\beta$-lyase activity and, thus, the use of the Metschnikowia pulcherrima NS-EM-34 (previously used in red wine production due to its remarkable pectinolytic activity; Belda et al. 2016c) to increase polyfunctional thiol release in wines has been explored in this work.

Thus, this work aims to study the sensorial impact of $M$. pulcherrima NS-EM-34 in sequential fermentations with two industrial strains of Saccharomyces cerevisiae and its specific influence on the varietal perception of Verdejo wines, measuring the production of minor (terpenes and thiols) and major varietal volatile compounds accompanied with sensory analyses of wines.

\section{Materials and methods}

\section{Yeast strains and molecular identification}

Two $S$, cerevisiae industrial strains obtained from Agrovin S.A. (Alcázar de San Juan. Spain) (S. cerevisiae Viniferm Diana and $S$. cerevisiae Viniferm Revelacion) and another pre-commercial strain $M$. pulcherrima NS-EM-34 (GenBank accession number KT222665: also deposited and publicly available in the Complutense Yeast Collection) were used in this work.

\section{Microvinifications and growth kinetics}

All fermentations in this study were carried out using the must from Vitis vinifera L.cv. Verdejo grapes processed as described by Benito et al. (2012). Fermentations were run in triplicate in 5-L glass bottles with air-lock system allowing carbon dioxide emission, using $3.5 \mathrm{~L}$ of débourbage fresh must. The composition of the must was: $223 \mathrm{~g} / \mathrm{L}$ sugar, $214 \mathrm{mg} / \mathrm{L}$ YAN (yeast assimilate nitrogen), $1.89 \mathrm{~g} / \mathrm{L}$ malic acid, and $\mathrm{pH} 3.31$. The must was frozen at $-30^{\circ} \mathrm{C}$ for 6 months to reduce indigenous flora (values under $10^{3}$ viable cells $/ \mathrm{mL}$ ). being defreezed before fermentations.

Four assays were carried out: (1) inoculation with $S$. cerevisiae Viniferm Revelacion (ScR), (2) inoculation with $S$. cerevisiae Viniferm Diana (ScD), (3) sequential inoculation with $M$. pulcherrima followed by $S$. cerevisiae Viniferm Revelacion (MP...ScR) after 15-g/L sugar consumption, and (4) sequential inoculation with $M$. pulcherrima followed by $S$. cerevisiae Viniferm Diana (MP...ScD) after $15-\mathrm{g} / \mathrm{L}$ sugar consumption. Fermentations were performed at $20^{\circ} \mathrm{C}$ without shaking.

All strains were grown for biomass production in an enriched must medium ( $12.5 \%$ concentrated must (final concentration: $50 \mathrm{~g} / \mathrm{L}$ glucose + fiuctose). $1 \%$ yeast extract, $0.5 \%$ proteose peptone no.3, pH 3.5) $48 \mathrm{~h} \mathrm{at} 25^{\circ} \mathrm{C}$. before their use in microvinifications. Cultures were adjusted to a final cell concentration of $10^{6}$ cells $/ \mathrm{mL}$. Aliquots were taken periodically during fermentations. Growth kinetics were followed by plating $50 \mu \mathrm{L}$ of the appropriate dilution on Sabouraud glucose agar with chloramphenicol (PanReac, Barcelona, Spain) and lysine media (Oxoid, Hampshire. UK), for total yeast counts and non-Saccharomyces counts, respectively. After $48-72 \mathrm{~h}$ at $28^{\circ} \mathrm{C}$, colonies were counted.

After glucose + fructose concentration fell to values lower than $4 \mathrm{~g} / \mathrm{L}$ (sugar fermentation completed). $50 \mathrm{mg} / \mathrm{L}$ of sulfur 
dioxide was added to the wine as potassium metabisulfite (PanReac, Barcelona, Spain). Then, the wines were racked and stabilized during 7 days at $4{ }^{\circ} \mathrm{C}$. After that, the final products were bottled in glass bottles with crown caps, with the minimum air chamber in the bottleneck. Until sensorial evaluation, the bottles were stored horizontally in a climate chamber TR2V120 (La Sommelière, Saint-Saturnin, France) at $18{ }^{\circ} \mathrm{C}$ and $70 \%$ relative humidity.

\section{Analytical determinations of non-volatile compounds}

The concentrations of glucose + fructose, acetaldehyde, acetic acid, L-malic acid, L-lactic acid, citric acid, and glycerol were determined using the Y15 Enzymatic Autoanalyzer (Biosystems S.A, Barcelona, Spain), with the appropriate kits supplied by the manufacturer. The alcohol content was determined by using the GAB Microebu boiling method (http://shop.gabsystem.com). The $\mathrm{pH}$ was measured with a Crison $\mathrm{pH}$ Meter Basic 20 (Crison, Barcelona, Spain).

\section{Analytical determinations of volatile compounds}

The analysis of volatile compounds in the final wines was performed by gas chromatography-mass spectrometry (GCMS), following the analytical approach previously described in Belda et al. (2017b). In summary, the method described by Rapp et al. (1994) was used to determine fermentation byproducts (major volatile compounds), with the slight modifications described in detail by Belda et al. (2017b). Due to an instrumental error, replicates 2 and 3 of Mp...ScR assay were not quantified for major volatile compounds.

For analyzing volatile thiols, the quantitative methodology described by Ferreira et al. (2007), later optimized by Kapaklis (2014), were applied, following the final protocol previously described in Belda et al. (2017b).

\section{Sensorial analysis}

Final wines were tested blindly by a panel of ten experienced wine tasters, all of them members of the staff of the Food Technology Department of the Polytechnic University of Madrid and Microbiology Department of the Complutense University of Madrid. The members evaluated the wines using 12 attributes previously described by consensus according to their idea of Verdejo wine tipicity, in two different sessions that took place on different days, testing all wines in triplicate. A scale from 0 (no character) to 10 (very strong character) was used to rate the intensity of the 12 attributes, according to the panelists criteria.

\section{Statistical analysis}

The statistical analyses were performed using PC Statgraphics v.5 Software (Graphics Software Systems, Rockville, MD, USA). The significance was set to $p<0.05$ for the ANOVA matrix F-value. Means were compared using the multiplerange test.

\section{Results}

\section{Population dynamics and sugar consumption}

S. cerevisiae fermentation ( $\mathrm{ScD}$ and $\mathrm{ScR}$ ) took 15 days to be complete and sequential fermentation with $M$. pulcherrima NS-EM-34 and $S$. cerevisiae (Mp...ScD and $\mathrm{Mp}$...ScR) took 26 days. Figure 1 shows total yeast counts and $M$. pulcherrima counts monitored during wine fermentation. Both $\mathrm{ScD}(\mathrm{A})$ and $\mathrm{ScR}(\mathrm{B})$ reached their highest counts at day 4 and presented very similar kinetics during the course of fermentation. Regarding sequential fermentations, where $S$. cerevisiae strains were inoculated at day 4 (when $15 \mathrm{~g} / \mathrm{L}$ of sugar consumption was reached), a similar behavior was observed. From this point, $M$. pulcherrima population started to decrease, ceasing to detect non-Saccharomyces yeasts after day 9 . Residual glucose and fructose (Fig. 1) decreased slightly until $S$. cerevisiae strains were inoculated; after $S$. cerevisiae inoculations, the sugar reduction quickly accelerated. At day 9, the maximum total count was reached in both fermentations, corresponding to $S$. cerevisiae exponential growth, showing a high cell viability until the end of fermentation. Both $\mathrm{Mp} \ldots \mathrm{ScD}$ and $\mathrm{Mp}$...ScR fermentations presented parallel kinetics, corresponding to the absence of an important difference between ScD and ScR population dynamics.

\section{Wine analytic parameters}

Table 1 shows the final concentration of different compounds representative of different fermentation parameters. Glucose + fructose final concentrations did not show statistically significant differences, while final ethanol concentrations were lower for the fermentations involving $M$. pulcherrima in about $0.6 \%(v / v)$. These fermentations (Mp...ScD and Mp...ScR) showed higher concentrations in glycerol that varied from 0.52 to $0.72 \mathrm{~g} / \mathrm{L}$.

Regarding the metabolism of acids, a slight malic acid degradation (varying from 0.1 to $0.2 \mathrm{~g} / \mathrm{L}$ ), but higher than for $S$. cerevisiae alone, took place in fermentations involving $M$. pulcherrima. Nevertheless, for this study, the malic acid reduction did not affect the final $\mathrm{pH}$. No statistical differences were observed for the final acetic acid concentration, which 
Table 1 Analytical results of fermentation parameters at the end of the different fermentation assays

\begin{tabular}{lllll}
\hline Compounds & $\mathrm{ScD}$ & $\mathrm{ScR}$ & $\mathrm{MP} \ldots \mathrm{ScD}$ & $\mathrm{MP} \ldots \mathrm{ScR}$ \\
\hline L-Lactic acid $(\mathrm{g} / \mathrm{L})$ & $0.10 \pm 0.01 \mathrm{a}$ & $0.10 \pm 0.01 \mathrm{a}$ & $0.14 \pm 0.03 \mathrm{a}$ & $0.15 \pm 0.05 \mathrm{a}$ \\
L-Malic acid $(\mathrm{g} / \mathrm{L})$ & $1.83 \pm 0.06 \mathrm{c}$ & $1.60 \pm 0.00 \mathrm{~b}$ & $1.62 \pm 0.06 \mathrm{ab}$ & $1.51 \pm 0.07 \mathrm{a}$ \\
Acetic acid $(\mathrm{g} / \mathrm{L})$ & $0.39 \pm 0.01 \mathrm{a}$ & $0.37 \pm 0.01 \mathrm{a}$ & $0.36 \pm 0.03 \mathrm{a}$ & $0.38 \pm 0.04 \mathrm{a}$ \\
Glucose + fructose $(\mathrm{g} / \mathrm{L})$ & $3.27 \pm 0.15 \mathrm{a}$ & $3.57 \pm 0.35 \mathrm{a}$ & $3.45 \pm 0.36 \mathrm{a}$ & $3.66 \pm 0.41 \mathrm{a}$ \\
Glycerol $(\mathrm{g} / \mathrm{L})$ & $4.76 \pm 0.05 \mathrm{a}$ & $5.00 \pm 0.07 \mathrm{~b}$ & $5.48 \pm 0.08 \mathrm{c}$ & $5.52 \pm 0.09 \mathrm{c}$ \\
pH & $3.36 \pm 0.03 \mathrm{ab}$ & $3.33 \pm 0.01 \mathrm{a}$ & $3.36 \pm 0.02 \mathrm{a}$ & $3.35 \pm 0.02 \mathrm{a}$ \\
Acetaldehyde $(\mathrm{mg} / \mathrm{L})$ & $54.67 \pm 5.13 \mathrm{~b}$ & $47.69 \pm 3.79 \mathrm{~b}$ & $39.00 \pm 2.11 \mathrm{a}$ & $37.42 \pm 3.28 \mathrm{a}$ \\
Citric acid $(\mathrm{g} / \mathrm{L})$ & $0.24 \pm 0.01 \mathrm{a}$ & $0.25 \pm 0.01 \mathrm{a}$ & $0.26 \pm 0.02 \mathrm{a}$ & $0.23 \pm 0.02 \mathrm{a}$ \\
Alcohol $(\% \mathrm{v} / \mathrm{v})$ & $13.00 \pm 0.01 \mathrm{~b}$ & $12.97 \pm 0.06 \mathrm{~b}$ & $12.38 \pm 0.05 \mathrm{a}$ & $12.34 \pm 0.04 \mathrm{a}$ \\
\hline
\end{tabular}

Results represent the mean with standard deviations (SD) for three replicates. Means in the same row with the same letter are not significantly different $(p<0.05)$

$S c D$ fermentation performed by inoculation with $S$. cerevisiae Viniferm Diana, $S c R$ fermentation performed by inoculation with $S$. cerevisiae Viniferm Revelacion, $M P$...ScD fermentation performed by sequential inoculation with $M$. pulcherrima followed by $S$. cerevisiae Viniferm Diana, $M P$...ScR fermentation performed by sequential inoculation with $M$. pulcherrima followed by $S$. cerevisiae Vinifem Revelacion varied in the biggest case from 0.36 to $0.39 \mathrm{~g} / \mathrm{L}(\mathrm{Mp} \ldots \mathrm{ScD}$ and $\mathrm{ScD}$, respectively).

Finally, the final concentration of acetaldehyde in fermentations involving $M$. pulcherrima was always lower than the correspondent single control fermentations, varying in $15.67 \mathrm{mg} / \mathrm{L}(\mathrm{Mp} \ldots \mathrm{ScD}$ compared with ScD) and $10.27 \mathrm{mg} /$ L (Mp...ScR compared with ScR).

\section{Major volatile compounds}

\section{Higher alcohols}

The final volatile compound concentration of the different wines used in this study is represented in Fig. 2. Although there are some statistically significant differences between the studied $S$. cerevisiae strains, sequential fermentations involving $M$. pulcherrima showed lower concentrations in all higher alcohols, except for 2phenylethan-1-ol (Supplemental Fig. S1; Supplemental Table S1). The average effect of $M$. pulcherrima in decreasing the total higher alcohol concentration was 72.46 $\pm 6.94 \mathrm{mg} / \mathrm{L}$. while the difference between $S$. cerevisiae strains implies: $34.76 \mathrm{mg} / \mathrm{L}$ (in $S$. cerevisiae single fermentation) and $24.95 \mathrm{mg} / \mathrm{L}$ (in sequential fermentation). The most significant differences between sequential and the control-single fermentations were observed in 3methyl-butan-1-ol and hexan-1-ol, reaching average values of decrease of $66.08 \mathrm{mg} / \mathrm{L}$ (about a 2 -fold decrease in sequential fermentations) and $1.11 \mathrm{mg} / \mathrm{L}$ (about a 3 -fold decrease in sequential fermentations). respectively.

Contrary, regarding 2-phenylethan-1-ol, $M$. pulcherrima produced a slight average increase of $5.43 \mathrm{mg} / \mathrm{L}(1.3$-fold increase) when compared with the control wines.

\section{Esters}

M. pulcherrima sequential fermentations reported slightly lower ester concentrations except for 2-phenylethtyl acetate. whose concentrations are related to the 2-phenylethan-1-ol concentration reported above (Supplemental Fig. S2; Supplemental Table $\$ 1$ ). Although there are statistically significant differences in total ester concentration between single and sequential fermentation (Fig. 2), the average effect in total ester concentrations of $M$. pulcherrina was very low. The highest levels observed for ethyl butanoate. ethyl hexanoate. and ethyl octanoate were related to $S$. cerevisiae Revelacion strain (both in single and sequential fermentations).

\section{Fatty acids}

A similar effect than in higher alcohols and esters took place for fatty acids. $M$. pulcherrima sequential fermentations showed slightly lower concentrations in the total concentration of volatile fatty acids than the control assays (Fig. 2). However, only the differences in pentanoic acid had statistical significance between conditions. A contrary effect occurs for octanoic acid, since $M$. pulcherrima increased the concentration (Supplemental Fig. S3: Supplemental Table S1).

\section{Varietal thiols.}

Fermentations where $M$. pulcherrima were involved showed lower final concentrations of 3-sulfanylhexan-1-ol (3-SH) than in the cases of $S$. cerevisiae pure fermentations, showing only statistical differences for ScR fermentation (Fig. 3). However, in all the assays. 3-SH concentration markedly reached its detection threshold $(60 \mathrm{ng} / \mathrm{L}$ ) (Swiegers et al. 
a

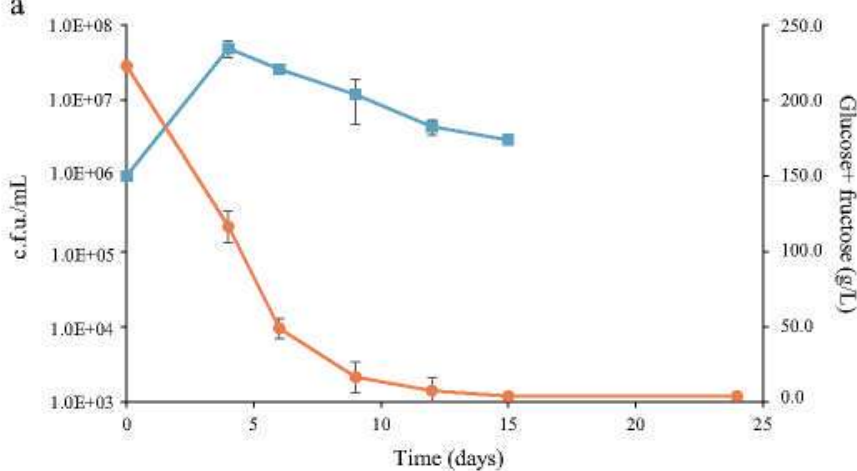

$\mathrm{c}$

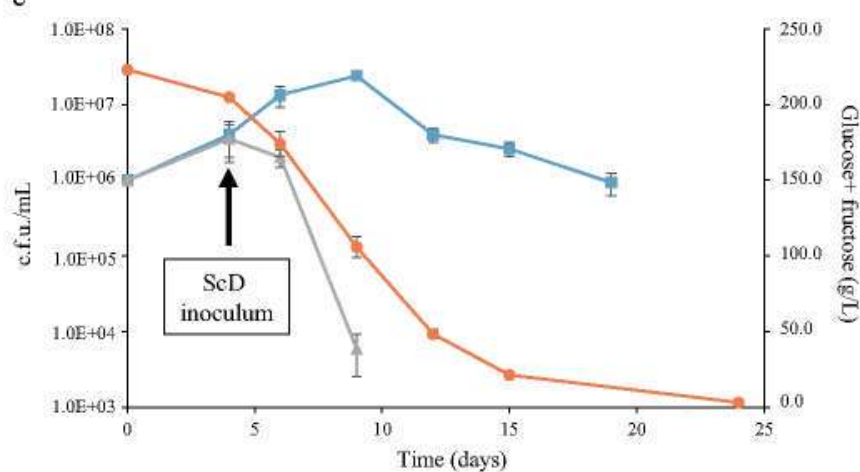

Fig. 1 Total yeast count (blue square) and $M$. pulcherrima NS-EM-34 (gray triangle) during fermentation. The residual glucose + fructose $(\mathrm{mg} /$ L) during fermentation (orange circle) is also represented. a Fermentation performed by inoculation with $S$. cerevisiae Viniferm Diana $(\mathrm{ScD})$. b Fermentation performed by inoculation with $S$. cerevisiae Viniferm Revelacion (ScR). c Fermentation performed by sequential inoculation

2005). There is also a significant decrease in the concentration of the 3-SH-derived compound 3-SHA.

The most significant difference took place for the final concentrations of 4-methyl-4-sulfanylpentan-2-one (4-MSP), showing a clear effect of $M$. pulcherrima metabolism (Fig. 3). S. cerevisiae fermentations produced 4-MSP at a concentration lightly upper of 4-MSP detection threshold (3 $\mathrm{ng} / \mathrm{L})$ (Swiegers et al. 2005), and only in the case of ScR fermentation (not detectable levels, in the case of $\mathrm{ScD}$ fermentation). On the contrary, both sequential fermentations with $M$. pulcherrima ( $\mathrm{Mp} \ldots \mathrm{ScD}$ and $\mathrm{Mp} \ldots \mathrm{ScR})$ produced 4-MSP at final concentrations 6.4-fold higher than ScR assay, reaching mean values of $28.05 \mathrm{ng} / \mathrm{L}$.

\section{Sensorial analysis.}

Fermentations involving $M$. pulcherrima showed higher scores in Verdejo typicity, aroma quality, aroma intensity, fruity, and overall impression (Supplemental Fig. S4). The higher concentration in 4-MSP and lower concentration reported in higher alcohols, esters, and fatty acids for $M$. pulcherrima fermentations, clearly influence the Verdejo varietal perception (whose main descriptors are related to varietal thiol character). Even the M. pulcherrima showed less total b

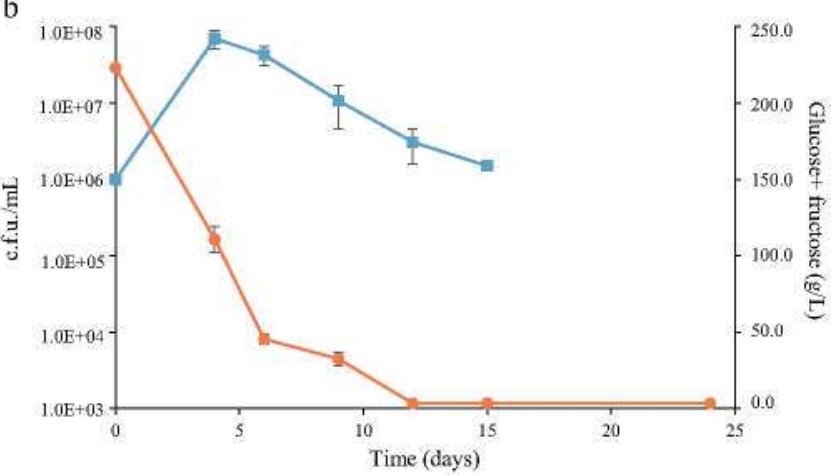

d

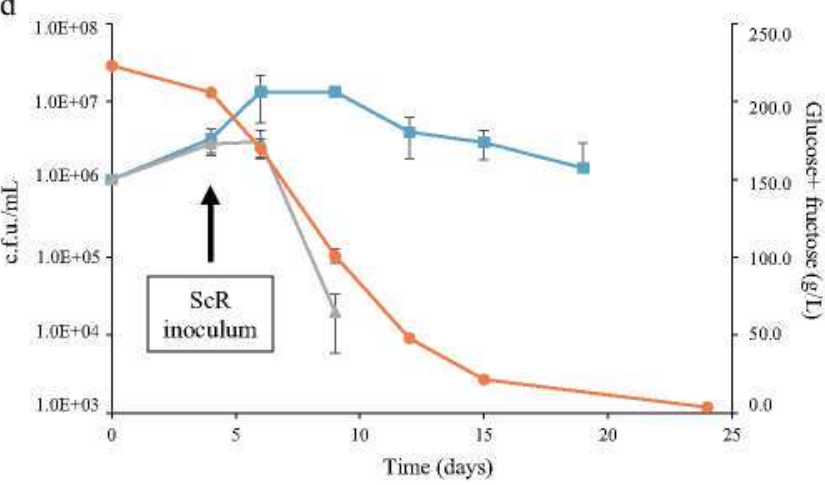

with $M$. pulcherrima followed by $S$. cerevisiae Viniferm Diana (MP... ScD). d Fermentation performed by sequential inoculation with $M$. pulcherrima followed by S. cerevisiae Viniferm Revelacion (MP... $\mathrm{ScR}$ ). Black arrow represents the moment of inoculation of the $S$. cerevisiae strain in sequential fermentation (after $15 \mathrm{~g} / \mathrm{L}$ of sugar consumption). c.fu. $=$ colony-forming units

volatile compounds, the small detection threshold of 4-MSP increased the perception of a higher aroma intensity.

\section{Discussion}

\section{Fermentation kinetics}

Both single S. cerevisiae fermentations ( $\mathrm{ScD}$ and $\mathrm{ScR}$ ) and the correspondent sequential trials with M. pulcherrima (Mp... $\mathrm{ScD}$ and $\mathrm{Mp}$...ScR) were able to finish the alcoholic fermentation, through a complete sugar depletion. Sequential inoculation allowed the $M$. pulcherrima population development during the first 4 days, which is necessary to observe the effect of this non-Saccharomyces species on fermentation. Mixed simultaneous inoculations of S. cerevisiae and M. pulcherrima did not permit enough M. pulcherrima development (Varela et al. 2017) limiting its influence on fermentation, as has been also described for other important non-Saccharomyces species such as Torulaspora delbueckii (Belda et al. 2015). Previous works using $M$. pulcherrima and $S$. cerevisiae, in white wine sequential fermentations, showed similar population kinetics, with a significant decrease of $M$. pulcherrima cell viability at, 
Fig. 2 Major volatile compound group quantified in the different fermentations performed in the study (represented in milligrams per liter). Different letters on the bars mean significant differences between assays $(p<0.05)$

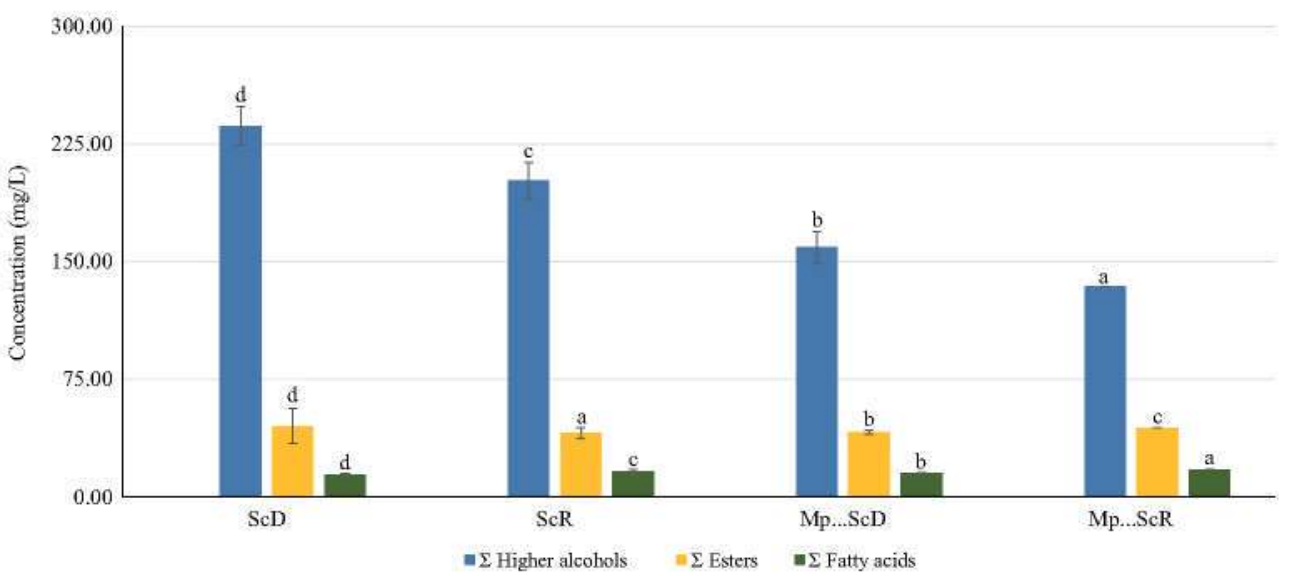

approximately, the day 9 of fermentation (García et al. 2017; Sadoudi et al. 2017).

Similar trends have been also observed attending to the sugar consumption rate (Beckner-Whitener et al. 2016) and other authors observed similar kinetics in weight loss that is related to sugar and fermentation metabolism (Barbosa et al. 2018). The slower sugar consumption showed that between days 0 and 4 in fermentations involving M. pulcherrima (Fig. 1) can be related to its moderate fermentation power compared with $S$. cerevisiae wine strains. Because of that, fermentations carried out with $M$. pulcherrima reached sugar depletion later than $S$. cerevisiae fermentations. This fact is also in concordance with other works using both the same $M$. pulcherrima strain in semi-industrial red wine fermentations (Belda et al. 2016c) and other strains (Sadoudi et al. 2017).

\section{Basic analytical profile of wines}

Starting with the metabolism of sugars, and taking into account the level of reduction in ethanol production observed in sequential fermentations (as a mean, $0.6 \%$ less in fermentations involving $M$. pulcherrima than in their correspondent single control fermentations), it should be mentioned that bigger differences have been observed by other authors, up to
$1.1 \%(v / v)$ (Varela et al. 2017), 0.8\% (v/v) (Sadouidi et al. Sadoudi et al. 2012), but also smaller differences of about $0.2 \%(v / v)$ (Benito et al. 2015), $0.28 \%(v / v)$ (Comitini et al. 2011), and $0.5 \%(v / v)$ (González-Royo et al. 2015), once again highlighting a notable strain- and condition-dependence for these subtle parameters. In the case of glycerol production, other studies reported increases in glycerol concentration of mixed cultures involving $M$. pulcherrima, varying from 0.3 to $0.79 \mathrm{~g} / \mathrm{L}$ depending on the $M$. pulcherrima proportion in the initial inoculum (Comitini et al. 2011). Other authors also reported notable increases of glycerol content in different white varieties in $M$. pulcherrima sequential fermentations, such as Riesling, with a mean increase of $0.24 \mathrm{~g} / \mathrm{L}$ (Benito et al. 2015), or Chardonnay, with a mean increase of $0.62 \mathrm{~g} / \mathrm{L}$, but that was not observed in the case of the red variety Shiraz (Varela et al. 2016). These increases in glycerol production, in the presence of $M$. pulcherrima, have been explained by the overexpression of GDPl gene in $S$. cerevisiae (involved in dihydroxyacetone phosphate conversion to glycerol-3-phosphate, intermediate for glycerol formation) that is overinduced during its co-existence with $M$. pulcherrima in must fermentations (Sadoudi et al. 2017).

Regarding the final concentration of acetic acid, other studies also reported no significant differences between $M$.
Fig. 3 Polyfunctional thiol release $(\mathrm{ng} / \mathrm{L})$ in the four different fermentation assays. 3SH: 3 sulfanylhexan-1-ol; 3SHA: 3 sulfanylhexyl acetate; 4MSP: 4methyl-4-sulfanylpentan-2-one. The mean SD for three replicates is shown. Different letters on the bars mean significant differences between assays $(p<0.05)$

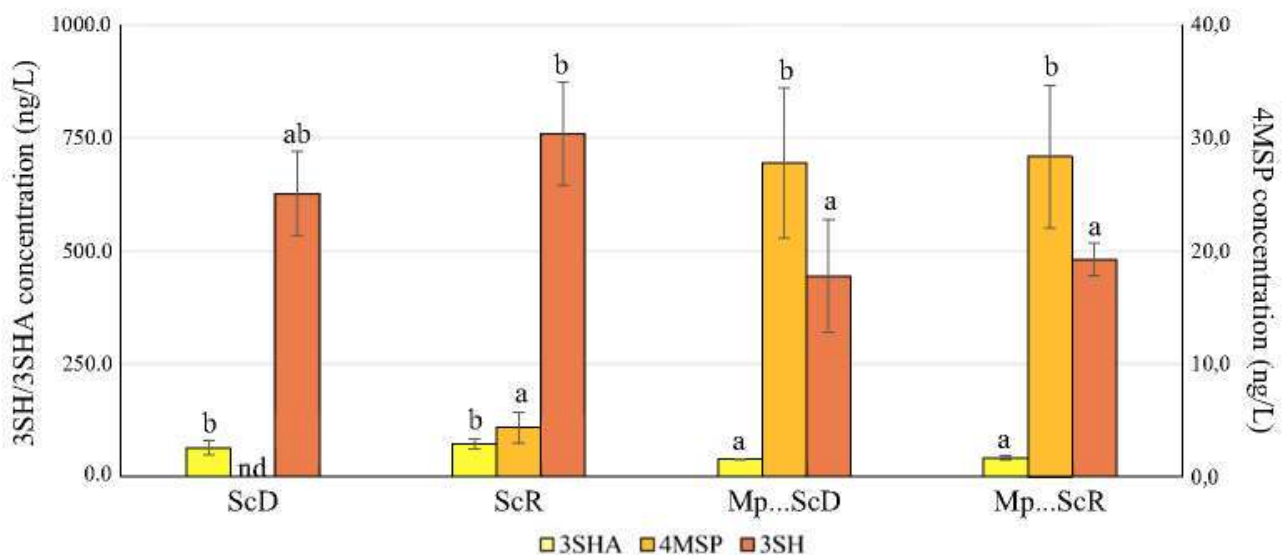


pulcherrima sequential fermentation and the $S$. cerevisiae controls (Benito et al. 2015; Chen et al. 2018; GonzálezRoyo et al. 2015). Other scientific works reported significant changes in the acetic acid content of sequential fermentations involving $M$. pulcherrima, reducing its final concentration in about $0.18 \mathrm{~g} / \mathrm{L}$ (Sadoudi et al. 2012) or $0.11 \mathrm{~g} / \mathrm{L}$ (Barbosa et al. 2018), and also drastically reducing it reaching final levels near to $0.06 \mathrm{~g} / \mathrm{L}$ (Contreras et al. 2015). However, other authors reported the opposite effect (Whitener et al. 2017), but in any case, with final concentrations remaining below the perception threshold. A study that compared $7 \mathrm{M}$. pulcherrima strains and $3 S$. cerevisiae strains in pure culture reported all $M$. pulcherrima strains to produce lower acetic acid concentrations (of about $0.5 \mathrm{~g} / \mathrm{L}$ ) than the studied $S$. cerevisiae strains (Comitini et al. 2011). The same study reports acetic acid reductions, in sequential fermentations, varying from 0.12 to $0.2 \mathrm{~g} / \mathrm{L}$. In the same line, regarding the metabolism of malic acid, previous studies reported similar results than those observed in this work, with slight degradations of about $0.1 \mathrm{~g} / \mathrm{L}$ (Whitener et al. 2017) or $0.3 \mathrm{~g} / \mathrm{L}$ (Chen et al. 2018). However, other authors reported the opposite effect with statistical increases varying from 0.11 to 0.86 , depending on the grape variety (Varela et al. 2016).

Finally, attending to acetaldehyde level in the final wines, as an undesirable compound (negatively impacting wine flavor, commonly associated by consumers with wine oxidation aromas), its significant reduction in sequential fermentations using $M$. pulcherrima is mentionable, as has been previously reported, in similar reduction values of about $20 \mathrm{mg} / \mathrm{L}$, by Benito et al. (2015).

\section{Volatile profile of wines (major compounds)}

Starting with the differences observed in the higher alcohol content of wines, it should be mentioned that lower concentrations of these compounds tend to be related with an improvement in wine aromatic complexity (Romano and Suzzi 1993). The reason of that has been explained in some sensorial works, concluding that, in general, the lower dominance of alcoholic compounds the better sensitivity to perceive the overall aroma expressed in wines (by a better perception of minority aroma compounds, such as the varietal fraction of wine aroma) (Frost et al. 2015).

In accordance with our results, previous studies reported similar effects of a $M$. pulcherrima wine strain decreasing the total amount of higher alcohols in wines (Benito et al. 2015). In the mentioned study, the use of $M$. pulcherrima FlaviaR in sequential fermentations decreased, as a mean, $47 \mathrm{mg} / \mathrm{L}$ in the total amount of higher alcohols compared with $S$. cerevisiae single inoculations (being $72.5 \mathrm{mg} / \mathrm{L}$, the analogous value reached in the present work). More specifically, our results totally agreed with those reported by Varela et al. (2016) in trials using Shiraz red variety, where all the higher alcohols showed significant decreases when M. pulcherrima was used, except for 2-phenylethan-1-ol, which showed a concentration increase of about $35 \mathrm{mg} / \mathrm{L}$. Previous studies (ClementeJiménez et al. 2004) also reported M. pulcherrima as a yeast species able to produce notable amounts of 2-phenylethan-1-ol.

Nevertheless, it should be mentioned that Sadoudi et al. (2012) observed an increase in the total content of higher alcohols in simultaneous mixed fermentations between $M$. pulcherrima and $S$. cerevisiae, while this was not observed for $M$. pulcherrima in pure culture inoculation. The same work also observed increases in 2-phenylethan-1-ol in all fermentations involving $M$. pulcherrima in pure and mixed inoculation.

Regarding the concentration of esters, some studies have reported contradictory results on the effect of $M$. pulcherrima on them. Contreras et al. (2014) showed 2-fold increases in the total ester concentration using $M$. pulcherrima in Chardonnay fermentations and a light decrease (1.1-fold decrease) in trials with Shiraz. Benito et al. (2015) reported an increase in ethyl octanoate by the sequential use of $M$. pulcherrima, when comparing to the $S$. cerevisiae control, up to $0.1 \mathrm{mg} / \mathrm{L}$. This fact has been also reported in other works (Clemente-Jimenez et al. Clemente-Jiménez et al. 2004; Lambrechts and Pretorius 2000) with notable increases in this pear-associated aroma by the action of $M$. pulcherrima. It should be mentioned that, during our work, a strong pear-like aroma was perceived in those assays involving M. pulcherrima, but it was only detectable at the first stages of the fermentations (when $M$. pulcherrima remains as the sole inoculum), disappearing through the alcoholic fermentation. Although a slight increase in ethyl octanoate was observed in this study with $M$. pulcherrima when combined with $S$. cerevisiae Diana (compared to the $\mathrm{ScD}$ control fermentation), the strongest effect in this case was observed from the $S$. cerevisiae Revelacion strain.

\section{Varietal thiols of wines}

The main volatile thiol compounds contributing to the varietal aroma fraction of white wines (such as Verdejo varietal wines) were analyzed. In recent years, some studies have determined the contribution of particular non-Saccharomyces yeasts to wine composition, showing that some strains can be used to improve thiol concentration in wines. Furthermore, Knight et al. (2018) suggest that the yeast diversity in spontaneous wine fermentations can be directly correlated with the final thiol concentrations in wine, highlighting the contribution of Saccharomyces uvarum strains on it.

In our work, a marked effect of $M$. pulcherrima has been observed, decreasing the concentration of 3-SH and its acetate ester 3-SHA, but notably increasing the concentration of 4MSP. Sadoudi et al. (2012) also observed a similar effect in 3SH and 3-SHA concentrations using the other $M$. pulcherrima 
strain (in mixed-simultaneous inoculation) but, in this case. the level of 4-MSP was also diminished. This difference between studies can be explained since the $M$. pulcherrima studied by Sadoudi and collaborators was not previously tested and selected for its ability to increase thiol concentrations. but in the case of $M$. pulcherrima NS-EM-34, a notable cystathionine- $\beta$-lyase activity has been reported (Belda et al. $2016 \mathrm{~b}$ ), which could be related with its great impact in 4-MSP production.

In sequential fermentations carried out with a $T$. delbrueckii wine strain, this yeast produced, as occurred with $M$. pulcherrima NS-EM-34, significant increases in the production of 4-MSP. causing this volatile thiol to be in concentrations above its threshold of perception (Belda et al. 2017b). However, and in the same way as for the present study of $M$. pulcherrima, the levels of 3-SH and 3-SHA were not increased, the reason why it can be indicated that the presence of non-Saccharomyces could influence. fundamentally, the metabolic pathways that lead to the release of 4-MSP. However, this trait has been reported to be strain dependant (Zott et al. 2011) since the use of a T. delbrueckii wine strain in Sauvignon blanc fermentations also increased the levels of 3SH and 3SHA (Renault et al. 2016). In fact, the last studies regarding $M$. pulcherrina describe $\beta$-lyase as one of the most discriminant features among the different studied strains (Barbosa et al. 2018).

The higher 4-MSP levels (that generally impart box tree aroma (Tominaga et al.Tominaga et al. 1995)) in fermentations involving $M$. pulcherrima. contribute to the higher perception of thiol Verdejo character reported (Supplemental Fig. S4). 3-SH and 3-SHA impart passionfruit, grapefruit, gooseberry, and guava aromas and mainly contribute to fruity impression in wines. There are not weighty differences between single and sequential fermentations regarding the concentration of 3-SH and 3-SHA; however, the sensorial tests performed in this study display a higher fiuity impression in fermentations involving $M$. pulcherrima. This fact could be explained by the lower higher alcohol concentration reached in sequential fermentation wines, which can increase the varietal aroma perception. Frost et al. (2015) reported that the higher alcoholic content of wines the lower sensorialperceivable complexity of the wines, with dominant alcoholic compounds tending to overshadow minority aromas. These authors demonstrated that light decreases in the alcohol content of wines induce a better sensitivity by perception of overall aroma expressed in wines. Other studies reported the effect of producing lower amounts of higher alcohols in the sensorial perception of varietal aromas (Belda et al. 2015).

These $M$. pulcherrima-dependent parameters (lower higher alcohol concentration and the notable increase in the aromaimpact 4-MSP thiol) can be the basis to explain the higher scores, given by the panel of tasters. for the attributes 'aroma quality', 'aroma intensity', and 'overall impression' to all the sequential fermentations where $M$. pulcherrima were involved.

In summary, from this work. we can conclude that the use of selected $M$. pulcherrima strains is a useful biological strategy to increase the aromatic quality of specific white wines (such as Verdejo, or other relatively similar varieties like Sauvignon blanc or Chardonnay), where the varietal fraction of the aroma is a precious market-driven aspect. That increase in the aroma quality of wines under sequential $M$. pulcherrima fermentations has been attributed to the formation of 4-MSP in levels 6.4-fold higher than those produced by the control $S$. cerevisiae single fermentations, together with a generalized decrease in the concentration of higher alcohols. Other secondary advantages observed in the use of the selected $M$. pulcherrima strain are the production of higher glycerol content. and lower final concentrations of ethanol and acetaldehyde.

Funding Funding for the research in this paper was provided by Agrovin S.A. under the framework of the CDTI-financed project IDI-20160102 and by Pago de Carraovejas S.L. CDTI-financed project IDI-20160750 (Centre for Industrial Technological Development. Spanish Ministry of Econony, Industry and Competitiveness, Spain).

\section{Compliance with ethical standards}

Ethical statement Ten experienced wine tasters were involved in the study.

Conflict of interest I. Belda is now working at Biome Makers Inc; $\mathrm{E}$. Navascués is employee of Agrovin S.A.; all other authors declare that they have no conflict of interest.

\section{References}

Barbosa C, Lage P, Esteves M. Chambel L, Mendes-Faia A. MendesFerreira A (2018) Molecular and phenotypic characterization of Metschnikowia pulcherrima strains from Douro wine region. Fermentation 4:1-19

Baumes R (2009) Wine aroma precursors. In: Moreno-Aribas MV, Polo MC (eds) Wine chemistry and biochemistry. Springer-Verlag, New York, pp 251-265

Beckner-Whitener ME, Stanstrup J, Panzeri V, Carlin S, Divol B, Du Toit $\mathrm{M}$, Vrhovsek U (2016) Untangling the wine metabolome by combining untargeted SPME GCXGC-TOF-MS and sensory analysis to profile sauvignon blanc co-fermented with seven different yeasts. Metabolomics 12:53

Belda I, Navascués E, Marquina D. Santos A. Calderón F. Benito S (2015) Dynamic analysis of physiological properties of Torulaspora delbruechii in wine fermentations and its incidence on wine quality. Appl Microbiol Biotechnol 99:1911-1922

Belda I, Ruiz J, Navascués E. Marquina D. Santos A (2016a) Improvement of aromatic thiol release through the selection of yeasts with increased $\beta$-lyase activity. Int J Food Microbiol 225:1-8

Belda I. Ruiz J. Alastruey-Izquierdo A. Navascués E. Marquina D. Santos A (2016b) Unraveling the enzymatic basis of wine "flavorome": a phylo-functional study of wine related yeast species. Front Microbiol 7:12 
Belda I, Conchillo LB, Ruiz J, Navascués E. Marquina D, Santos A (2016c) Selection and use of pectinolytic yeasts for improving clarification and phenolic extraction in winemaking. Int J Food Microbiol 223:1-8

Belda I, Ruiz J. Esteban-Fernández A. Navascués E, Marquina D, Santos A. Moreno-Arribas MV (2017a) Microbial contribution to wine aroma and its intended use for wine quality improvement. Molecules 22:189-218

Belda I, Ruiz J, Besiert B. Navascués E. Marquina D, Calderón F, Rauhut D. Benito S, Santos A (2017b) Influence of Torulaspora delbruechii in varietal thiol (3-SH and 4-MSP) release in wine sequential fermentation. Int J Food Microbiol 257:183-191

Benito S, Palomero F. Morata A, Calderón F, Suárez-Lepe JA (2012) New applications for Schizosuccharomvces pombe in the alcoholic fermentation of red wines. Int J Food Sci Technol 47:2101-2108

Benito S, Hofnann T, Laier M. Lochbühler B. Schüttler A. Ebert K. Fritsch S. Röcker J, Rauhut D (2015) Effect on quality and composition of Riesling wines fermented by sequential inoculation with non-Saccharomyces and Saccharomvces cerevisiae. Eur Food Res Technol 241:707-717

Chen K, Escott C. Loira I, del Fresno JM, Morata A, Tesfaye W, Calderon F, Suárez-Lepe JA, Han S. Benito S (2018) Use of nonSaccharomvces yeasts and oenological tannin in red winemaking: influence on colour, aroma and sensorial properties of young wines. Food Microbiol 69:51-63

Clemente-Jiménez JF, Mingorance-Cazorla L, Martínez-Rodriguez S, Las Heras-Vázquez FJ, Rodríguez-Vico F (2004) Molecular characterization and oenological properties of wine yeasts isolated during spontaneous fermentation of six varieties of grape must. Food Microbiol 21:149-155

Conitini F, Gobbj M. Donizio P, Romani C. Lencioni L, Mannazzu I. Ciani M (2011) Selected non-Saccharomvces wine yeasts in controlled mutistarter fermentations with Saccharomvces cerevisiae. Food Microbiol 28:873-882

Contreras A, Hidalgo C. Henschke PA, Chambers PJ, Curtin C, Varela C (2014) Evaluation of non-Saccharomyces yeasts for the reduction of alcohol content in wine. Appl Environ Microbiol 80:1670-1678

Contreras A, Curtin C, Varela C (2015) Yeast population dynamics reveal a potential 'collaboration' between Metschnikowia pulcherrima and Saccharomyces unarum for the production of reduced alcohol wines during shiraz fermentation. Appl Microbiol Biotechnol 99:11851895

Dubourdieu D, Tominaga T, Masneuf I, des Gachons CP, Murat ML (2006) The role of yeasts in grape flavor development duing fermentation: the example of sauvignon blanc. Am J Enol Vitic 57:8188

Fedrizzi B. Pardon KH, Sefton MA. Elsey GM, Jeffery DW (2009) First identification of 4-\$-glutathionyl-4-methylpentan-2-one, a potential precursor of 4-mercapto- 4-methylpentan-2-one, in sauvignon blanc juice. J Agric Food Chem 57:991-995

Ferreira V, Ontin N, Cacho JF (2007) Optimization of a procedure for the selective isolation of some powerful aroma thiols. Development and validation of a quantitative method for the determination in wine. $\mathrm{J}$ Chromatogr A 1143:190-198

Frost R. Quiñones I, Veldhuizen M, Alava JI. Small D. Carreiras M (2015) What can the brain teach us about winemaking? An fMRI study of akohol level preferences. PLoS One 10:e119220

Garcia M. Esteve-Zarzoso B. Crespo J, Cabellos JM. Arroyo T (2017) Yeast monitoring of wine mixed or sequential fermentations made by native strains from D.O. "Vinos de Madrid" using real-time quantitative PCR. Front Microbiol 8:2520

González-Royo E, Pascual O, Kontoudakis N. Esteruelas M. EsteveZarzoso B. Mas A. Canals JM. Zamora F (2015) Oenological consequences of sequential inoculation with non-Saccharomyces yeasts (Tondaspora delbruechij or Metschnikowia pulcherrima) and Sactharomyces cerevisiae in base wine for sparkling wine production. Eur Food Res Technol 240:999-1012

Kapaklis A (2014) Impact of specific volatile thiols on varietal aroma of wines produced from Greek and some intemational grape varieties. $\mathrm{Ph}$.D. thesis, Justus- Liebig-University Gießen. Germany, URN: un: nbn: de: hebis: 26- opus-122144. http:/geb.uni-giessen.de/ geb/volltexte/2016/12214/

Knight SJ, Klaere S, Morrison-Whittle P. Goddard MR (2018) Fungal diversity during fermentation correlates with thiol concentration in wine. Aust J Grape Wine Res 24:105-112

Lambrechts MG. Pretorius IS (2000) Yeast and its importance to wine aroma. A review. S Afr J Enol Vitic 21:97-129

Mateo JJ, Jíménez M (2000) Monoterpenes in grape juice and wines. J Chromatogr A 881:557-567

Rapp A, Yavas I. Hastrich U (1994) Einfache und schnelle Anreicherung (Kaltronmethode) von Aromastoffen des Weines und deren quantitative Bestimnung mittels Kapillargaschromatographie. Deutsche Lebensmittel-Rundschau 90:171-174

Renault P. Coulon J, Moine V. Thibon C. Bely M (2016) Enhanced 3sulfanylhexan-l-ol production in sequential mixed fermentation with Torulaspora delbruechi/Saccharomyces cerevisiae reveals a situation of synergistic interaction between two industrial strains. Front Mícrobiol 7:293

Romano P. Suzzi G (1993) Higher alcohol and acetoin production by Zygosaccharomvces wine yeasts. J Appl Bacteriol 75:541-545

Sadoudi M. Tourdot-Maréchal R, Rousseaux S. Steyer D, GallardoChacón $\mathbf{J}$, Ballester $J$, Vichi $\$$, Guerin-Schneider $R$, Caixach $J$, Alexandre $\mathrm{H}$ (2012) Yeast-yeast interactions revealed by aromatic profile analysis of sauvignon blanc wine fermented by single or coculture of non-Saccharompces and Saccharomvces yeasts. Food Microbiol 32:243-253

Sadoudi M. Rousseaux S. David V. Alexandre H, Tourdot-Maréchal R (2017) Metschnikowia pulcherrima influences the expression of genes involved in PDH bypass and glyceropyruvic fermentation in Saccharomyces cerevisiae. Front Microbiol 8:1137

Swiegers H. Bartowsky E. Henschke P. Pretorius I (2005) Yeast and bacterial modulation of wine aroma and flavow. Aust J Grape Wine Res 11:139-173

Tominaga T, Masneuf I, Dubourdieu D (1995) A S-cysteine conjugate precursor of aroma of white sauvignon. J Int Sci Vigne Vin 29:227232

Tominaga T, Furer A, Henry R, Dubourdieu D (1998) Identification of new volatile thiols in aroma of Vitis vinifera $\mathrm{L}$. var. sauvignon blanc wines. Flavou Frag J 13:159-162

Varela C. Sengler F. Solomon M. Curtin C (2016) Volatile flavour profile of reduced alcohol wines fermented with the non-conventional yeast species Metschnikowia pulcherrima and Sactharomvces uvarum. Food Chem 209:57-64

Varela C, Barker A, Tran T, Borneman A. Curtin C (2017) Sensory profile and volatile aroma composition of reduced alcohol merlot wines fermented with Metschnikowia pulcherrima and Saccharomyces uvarum. Int J Food Microbiol 252:1-9

Whitener MEB, Stanstrup J, Carlin S, Divol B. Du Toit M, Vrhovsek U (2017) Effect of non-Saccharomyces yeasts on the volatile chemical profile of shiraz wine. Aust J Grape Wine Res 23:179-192

Zott K. Thibon C. Bely M, Lonvaud-Funel A. Duboudieu D. MasneufPonarede $I$ (2011) The grape nust non-Saccharomvces microbial conmunity: impact on volatile thiol release. Int J Food Microbiol $151: 210-215$ 